PROCEEDINGS OF THE

AMERICAN MATHEMATICAL SOCIETY

Volume 126, Number 1, January 1998, Pages 221-224

S $0002-9939(98) 04047-7$

\title{
AVERAGE CURVATURE OF CONVEX CURVES IN $H^{2}$
}

\author{
MARTIN BRIDGEMAN
}

(Communicated by Ronald A. Fintushel)

\begin{abstract}
A well-known result states that, if a curve $\alpha$ in $H^{2}$ has geodesic curvature less than or equal to one at every point, then $\alpha$ is embedded. The converse is obviously not true, but the embeddedness of a curve does give information about the curvature. We prove that, if $\alpha$ is a convex embedded curve in $H^{2}$, then the average curvature (curvature per unit length) of $\alpha$, denoted $K(\alpha)$, satisfies $K(\alpha) \leq 1$. This bound on the average curvature is tight as $K(\alpha)=1$ for $\alpha$ a horocycle.
\end{abstract}

\section{INTRODUCTION}

In the Euclidean plane the curves of constant curvature are circles and the curvature of a circle of radius $r$ is $1 / r$. Therefore as the radius of a circle increases the curvature decreases to zero. In the hyperbolic plane, as the radius of a circle increases the curvature decreases to one. It is a well-known fact that if a curve in $H^{2}$ has pointwise curvature less than or equal to one, then the curve is embedded in $H^{2}$. In this paper we study the converse: what does knowing that a curve is embedded in $H^{2}$ tell us about its curvature? We define the average curvature of a curve and show that if a convex curve is embedded in $H^{2}$, then its average curvature is bounded by one. The proof is an application of the isoperimetric inequality and the Gauss-Bonnett theorem.

I would like to thank Loyola University and I.H.E.S. for their support. Also I would like to thank Bill Thurston, Joe Christie, Curt McMullen and Rich Schwartz for many helpful conversations.

\section{BACKGROUND}

Using elementary hyperbolic geometry we can show that a circle or radius $r$ in $H^{2}$ has circumference given by $L(r)=2 \pi \sinh (r)$ and area by $A(r)=2 \pi(\cosh (r)-1)$.

By symmetry it is obvious that a circle of radius $r$ in $H^{2}$ has constant geodesic curvature given by some function $k(r)$. To find $k(r)$ we use the Gauss-Bonnett theorem.

Received by the editors June 13, 1996.

1991 Mathematics Subject Classification. Primary 51M09, 52A55; Secondary 52A38, 52A15.

(C)1998 American Mathematical Society 
Theorem 1 ([S]). Let $M$ be a two-dimensional Riemannian manifold and $S$ be a two-dimensional sub-manifold of $M$ with boundary $\partial S$. Then

$$
\int_{S} K \cdot d A+\int_{\partial S} k \cdot d s=2 \pi \chi(S)
$$

where $K$ is the gaussian curvature on $M$ and $k$ is the geodesic curvature of $\partial S$.

Thus applying this to the disk of radius $r$ in $H^{2}$ we get

$$
-1 \cdot A(r)+L(r) \cdot k(r)=2 \pi \cdot 1 .
$$

Substituting for $L$ and $A$ we get

$$
k(r)=\frac{\cosh (r)}{\sinh (r)}
$$

where $k$ is monotonically decreasing in $r$ with $\lim _{r \rightarrow \infty} k(r)=1$. A horosphere in $H^{2}$ can be considered a circle of radius infinity and it can be shown to have constant geodesic curvature one. Therefore we extend $k$ by defining $k(\infty)=1$.

In the Euclidean plane all curves with non-zero constant geodesic curvature are closed circles. In the hyperbolic plane it is very different. Curves with constant geodesic curvature greater than one are closed and curves of constant geodesic curvature less than or equal to one are embedded. The following well-known lemma gives a more general result about curves in $H^{2}$.

Lemma 1. If $\alpha$ is a curve in $H^{2}$ parameterized by arc length $s$ and geodesic curvature satisfying $k(\alpha(s)) \leq 1 \forall s \in R$, then $\alpha$ is embedded in $H^{2}$.

Thus if a curve has geodesic curvature less than or equal to one at every point, then it cannot intersect itself. The converse is obviously not true but we will show that if a curve is embedded, then we obtain a condition on its geodesic curvature.

\section{Average curvature}

Let $\alpha$ be a curve of finite length in $H^{2}$.

Definition 1. The average curvature of $\alpha$, denoted $K(\alpha)$, is given by

$$
K(\alpha)=\frac{\int_{\alpha} k(\alpha) d s}{\int_{\alpha}\left|\alpha^{\prime}\right| d s}=\frac{\text { Total curvature along } \alpha}{\text { Length of } \alpha}
$$

where $s$ is arc length along $\alpha . K(\alpha)$ is the geodesic curvature per unit length.

Obviously for $\alpha$ a circle of radius $r$ then $K(\alpha)=k(r)$.

If $\alpha$ is an infinite length curve we can still define $K(\alpha)$ by

$$
K(\alpha)=\varlimsup_{L \rightarrow \infty}\{K(\bar{\alpha}) \mid \bar{\alpha} \text { is a subarc of } \alpha \text { of length } L\} .
$$

A curve $\alpha$ is convex if any geodesic joining two points of $\alpha$ intersects $\alpha$ only at those two points. In the following we will restrict ourselves to convex curves in $H^{2}$. This restriction is necessary as embedded curves with arbitrarily large curvature can be constructed by spiralling between two circles (Figure 1). In general a curve with constant sign geodesic curvature either spirals or is convex.

Theorem 2. If $\alpha$ is a convex curve of length $L$ in $H^{2}$, then $K(\alpha) \leq F(L)$ where $F$ is monotonically decreasing in $L$ with $\lim _{L \rightarrow \infty} F(L)=1$.

Corollory 1. If $\alpha$ is a bi-infinite convex curve embedded in $H^{2}$, then $K(\alpha) \leq 1$. 


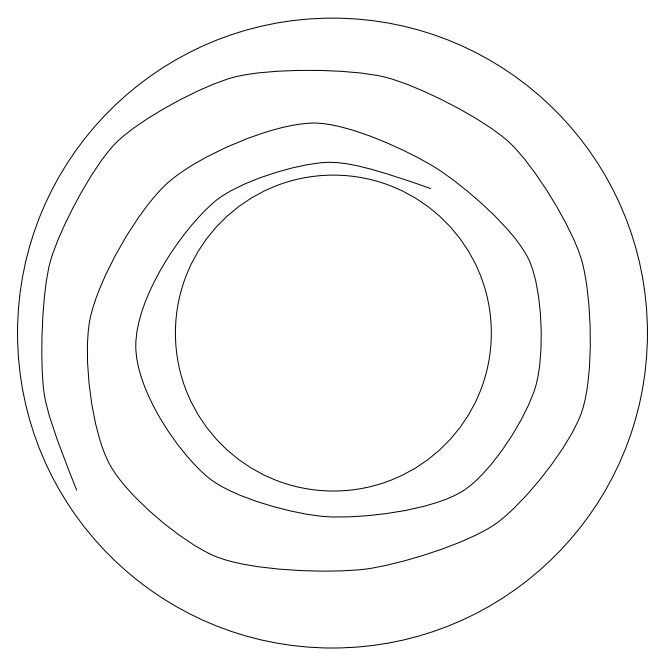

Figure 1. Spiralling curve

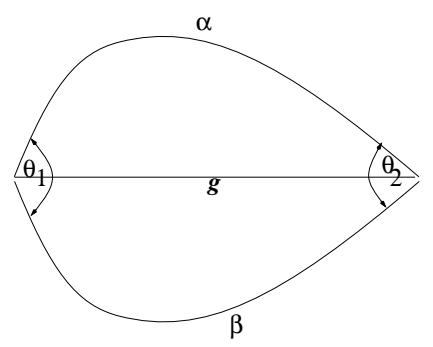

FiguRE 2. Curve $\gamma$

As a horosphere has average curvature equal to one, the above bound on average curvature is tight.

Proof of Theorem 2. From the above, we know that a circle of radius $r$ has circumference $L(r)=2 \pi \sinh (r)$, area $A(r)=2 \pi(\cosh (r)-1)$ and constant geodesic curvature $k(r)=\cosh (r) / \sinh (r)$. Rewriting the area and geodesic curvature in terms of the circumference $L$ we get

$$
k(L)=\sqrt{1+\left(\frac{2 \pi}{L}\right)^{2}} \quad \text { and } \quad A(L)=L \cdot k(L)-2 \pi .
$$

Now let

$$
F(L)=k(2 L)+\frac{\pi}{L}
$$

From the above, $F$ is monotonically decreasing in $L$ with $\lim _{L \rightarrow \infty} F(L)=1$.

Let $\alpha$ be a convex curve of length $L$ in $H^{2}$ and assume $K(\alpha)>F(L)$. Join the endpoints $x_{1}, x_{2}$ of $\alpha$ by a geodesic $g$. Reflect $\alpha$ in $g$ to get another curve $\beta$. Let $\gamma$ be the closed curve $\alpha \cup \beta$ and $\gamma^{*}$ be the disk bounded by $\gamma$. Label the interior angles of $\gamma^{*}$ at $x_{1}, x_{2}$ by $\theta_{1}, \theta_{2}$ respectively (Figure 2). Applying the Gauss-Bonnett 
theorem to $\gamma^{*}$ we get

$$
\begin{aligned}
& \int_{\gamma^{*}} K \cdot d A+\int_{\gamma} k \cdot d s=2 \pi \chi\left(\gamma^{*}\right) \\
& \Rightarrow-A+2 \int_{\alpha} k \cdot d s+\left(\pi-\theta_{1}\right)+\left(\pi-\theta_{2}\right)=2 \pi \\
& \Rightarrow A=2 L \cdot K(\alpha)-\theta_{1}-\theta_{2}
\end{aligned}
$$

where $A$ is the area bounded by $\gamma^{*}$.

Since $K(\alpha)>F(L)$ we have that

$$
\begin{gathered}
A>2 L\left(k(2 L)+\frac{\pi}{L}\right)-\theta_{1}-\theta_{2} \\
\Rightarrow A>2 L \cdot k(2 L)+2 \pi-\theta_{1}-\theta_{2} \geq 2 L \cdot k(2 L)-2 \pi,
\end{gathered}
$$

since both $\theta_{1}, \theta_{2} \leq 2 \pi$. Therefore

$$
\begin{gathered}
A>2 L \cdot k(2 L)-2 \pi=A(2 L) \\
\Rightarrow A>A(2 L) .
\end{gathered}
$$

Thus the curve $\gamma$ has length $2 L$ and bounds an area greater than the circle of the same length. This obviously contradicts the isoperimetric inequality which states that the maximum area inscribed by a curve of length $L$ is achieved only by a circle of perimeter $L$. Therefore $K(\alpha) \leq F(L)$.

Proof of Corollary 1. Let $\alpha$ be a bi-infinite convex curve embedded in $H^{2}$. Then for any subarc $\bar{\alpha}$ of $\alpha$ of length $L$ we have $K(\bar{\alpha}) \leq F(L)$. Therefore

$$
K(\alpha)=\varlimsup_{L \rightarrow \infty}\{K(\bar{\alpha}) \mid \bar{\alpha} \text { is a subarc of } \alpha \text { of length } L\} \leq \lim _{L \rightarrow \infty} F(L)=1 .
$$

Therefore $K(\alpha) \leq 1$.

\section{Conclusion}

For general convex curves the average curvature gives us a quantitative description of the general curvature of the curve. This can be extended to describe the average bending of a convex pleated plane in $H^{3}$. The author has applied this ([B]) to study the boundary of the convex core of a Kleinian manifold and limits of Kleinian groups.

\section{REFERENCES}

[B] M. Bridgeman, Average bending of boundaries of convex cores, In preparation.

[S] M. Spivak, A Comprehensive Introduction to Differential Geometry, Volume III, Publish or Perish (1979). MR 82g:53003c

Department of Mathematics, Loyola University, 6363 St. Charles Ave., New OrLEANS, LOUISIANA 70118

E-mail address: bridgemn@beta.loyno.edu 